the preseni and foreign things serve China', we will learn from the people of all countries. Like Copernicus, we will have the courage to break paths where none have gone before and to scale heights yet unclimbed. We will strive to catch up with and surpass the advanced world level in the near future to make a greater contribution to humanity."

\section{Paradox of Earth's core resolved}

from our Geomagnetism Correspondent

Althougr convection in the Earth's mantile is now widely accepted among geophysicists as a strong working hypothesis, it took a long time for this happy state of affairs to be reached from the original proposal of the concept. By contrast, the credibility of convection in the outer core has always seemed much greater and less likely to be eroded by expressions of doubt. To a large extent this is because the fluidity of the outer core was demonstrated by seismology many decades ago, whereas the acceptance of mantle convection requires belief in the more difficult and recent concept of plastic or semifluid flow in a solid or near-solid. But core convection also gained credibility by the necessity of explaining the origin of the geomagnetic field. The need for mantle convection depends on the acceptance of continental drift and seafloor spreading, acceptance which was gained only with a struggle and is even now not quite universal. The existence of the Earth's magnetic field, on the other hand, has never been in doubt, and an origin other than core convection has never proved viable.

Of course, core convection is still an article of faith rather than a proven phenomenon because the existence of fluid, rotating or not, does not in itself imply the presence of convection. Even so, geomagneticians have been somewhat dismayed recently to find Higgins and Kennedy (J. geophys. Res., 76, 1870; $1971)$ and Kennedy and Higgins ( $J$. geophys. Res., 78, 900; 1973) purporting to show that the core is stable against thermal convection. For convection to oecur in the core, the adiabatic temperature gradient throughout must be less than the melting temperature gradient, for if this is not the case the required instability cannot develop. Moreover, neither can there be convcction if the actual temperature is above the melting temperature and the actual temperature gradient is less than the adiabatic gradient, for convection is then less effective than conduction as a heat transfer mechanism. That the actual temperature throughout the outer core is indeed at or above the melting tem- perature can hardly be in doubt because the outer core is fluid. It is thus usual to assume that the actual temperature is higher than the melting temperature everywhere except at the inner coreouter core boundary where the temperatures are equal, supposing the boundary to represent the solid-liquid phase change of the core material.

If convection occurs, and because it is a very efficient heat transfer process, the actual temperature at all points will be very close to the adiabatic temperature -and the situation generally assumed hitherto is that both of these curves lie above the melting point curve. Higgins and Kennedy, on the other hand, revised the melting temperature of pure iron (the supposed principal core constituent) and the adiabatic temperature throughout the core, and concluded that the adiabatic temperature curve lies below the melting point curve. The core is still fluid, of course, and so the actual temperature curve is still above the melting temperature curve. But now there is no convection, and the actual and adiabatic temperature curves lie on opposite sides of the melting point curve.

It would be wrong to suggest that geomagneticians have been seriously concerned about all this; so sure are they of the reality of corc convection that they have generally been content to sit back and wait for someone to point out the flaw in the Higgins-Kennedy argument. Their wish has now apparently been granted by Frazer (Geophys. J., 34, $193 ; 1973)$. As Frazer points out, Higgins and Kennedy are on shaky ground right from the start because their melting curve is for pure iron, whereas the core almost certainly contains a proportion of lighter elements. The precise effects of these lighter constituents arc not known except that they are certain to decrease the melting temperature; but in any case Birch (Geophys. J., 29, 373; 1972) has concluded that in the present state of knowledge the melting temperature of iron at core pressures cannot be given with an accuracy greater than \pm $500 \mathrm{~K}$.

Higgins and Kennedy are thus less secure than they might seem because of the old and common problem of uncertainty in core parameters. But Frazer goes much further in elaiming that the Higgins-Kennedy argument is not valid even if their melting temperature curve is accepted because the assumptions made in their calculation of the adiabatic gradient are demonstrably inappropriate to the core; for example, one of the two methods used by Higgins and Kennedy involved equations derived by Valle (Annali Geofis., 5, 41; 1952). Strictly, these equations apply to a solid, but Valle supposed that at very high pressures a liquid behaves sufficiently like a solid for the same theory to be ap- plicable. At the same time, however, he supposed that the core behaves like a liquid in that the shear wave velocity is zero. The assumptions are thus physically incompatible-a defect which, Frazer claims, invalidates the theory's application to the core unless some more convincing justification can be given. Similar claims are made against the second method (involving Gruneisen's parameter $\Gamma$ ) uscd by Higgins and Kennedy.

But if Frazer's refutation of the case against convection put forward by Higgins and Kennedy is valid, it cannot necessarily be taken that convection actually occurs. Even if the simplifying assumptions adopted by Higgins and Kennedy are avoided, integration of the more general equation for the adiabatic gradient is still critically dependerit upon obtaining accurate values of the core's coefficient of thermal expansion $(\alpha)$ and specific heat at constant pressure $\left(C_{\mathrm{p}}\right)$. In fact, accurate values of these parameters are not known. It turns out that comparatively small changes in the $\alpha / C_{\mathrm{p}}$ ratio which are well within the present limits of uncertainty are sufficient to place the adiabatic temperature curve well above or well below the melting temperature curve. According to Frazer, it is thus not possible to say whether convection in the core is feasible or not. To that extent the convection implied by the existence of the geomagnetic field remains but an article of faith.

\section{Heterogeneous nuclear and messenger RNA}

\section{from ous}

Molecular Genetics Correspondent

ONF of the most important characteristics of messenger RNA synthesis in cukaryotic cells is the processing system in the nucleus which cleaves the mature messenger molecule from its much larger precursor. Because only a small proportion of the heterogeneous nuclear RNA (HnRNA) comprising the precursor is utilised to produce messengers-most of it is rapidly degraded within the nucleus-the maturation process represents a critical step in gene expression in eukaryotes. Three articles in the first issue of Cell are devoted to intriguing questions raised by the relation of $\mathrm{HnRNA}$ and $\mathrm{mRNA}$.

$\Lambda$ vital step in processing the large HnRNA to the much (up to ten times) smaller mRNA is the addition of a sequence of polyadenylic acid, probably one base at a time, to the $3^{\prime}$ end of the HnRNA molecule. This is then cleaved to generate the poly $(\Lambda)$-containing mINA. In eukaryotic nuclei, the length of this poly (A) is usually about 200 bases and inhibition of its addition prevents messenger production. 